\title{
A Technique to Improve Freehand Sketches of Multi-touch Interactions
}

\author{
Gil Barros, Leandro Velloso, and Luis Carli \\ FAU-USP: Faculty of Architecture and Urbanism, University of São Paulo \\ Rua do Lago, 876 - 05508.080 - São Paulo - SP - Brazil \\ \{gil.barros, leandrovelloso\}@usp.br, info@luiscarli.com
}

\begin{abstract}
Sketching is a core activity in design, but sketching practices in interaction design are sometimes insufficient, especially in the case of freehand sketching. In this article we indicate how a technique developed originally for the WIMP paradigm was extended to include multi-touch interfaces. We describe the technique, provide examples and show some future directions.
\end{abstract}

Keywords: Sketching, multi-touch, freehand, technique, ActionSketch.

\section{Introduction}

Sketching is a core activity in design [8] with great importance during early stages of the design process [11]. It is a well-established practice in many areas of design but is still in its infancy in the field of interaction design [5]. The field poses particular challenges for sketching and so current sketching practices are sometimes insufficient [5].

Several studies address this issue [7], but we focused on a particular approach: sketches as freehand drawings on paper. A review of the literature shows that it is still unexplored, despite being usually adopted and fostered as good practice $[6,10]$.

In previous works we proposed a technique to improve the process of sketching for interaction design [4]. The technique was designed for the WIMP paradigm (Windows, Icons, Menus, Pointer) and focused on rich interactions. We found that the technique improved the representation of the interaction on sketches and had positive effects in terms of the cognitive process of the designers and in the communication between professionals.

In this article we propose an extension to the technique to encompass its use for multi-touch interfaces, focusing particularly on tablets and smartphones.

\section{Extending the Technique}

Our first step was to understand the specific needs for the touchscreen environment. We analyzed the underlying technology by looking at available APIs for 
touchscreen $[2,3]$ as well as articles which proposed new forms of multi-touch interfaces $[1,12]$.

Based on the particularities found on this material, such as new events and parameters, we selected several multi-touch interactions, mostly from native applications of the iOS system, such as Safari, Mail, Weather and Springboard (Home Screen).

We then used our technique to represent these interactions, in order to compare our technique with the needs of multi-touch interfaces and to verify how it should be changed. From this comparison we found that the technique only needed minor extensions to be used with multi-touch interfaces. We now present the extended version of the technique, indicating the changes made in this version.

\section{The Technique: Frames, Colors, Symbols and Rules}

Frames are used to represent the interaction happening over time, arranged as a storyboard. This is a common practice in interaction design [5] and we found it to be the suitable for our technique.

Colors are applied to represent steps of the interaction. Each frame can have up to three steps: initial state, user actions and system actions. In order to indicate these steps in the same frame we use colors to separate them, following this association: initial state in black; user actions in green; system actions in orange.

Symbols are defined to represent basic user actions and responses from the system. The WIMP version of the technique has 12 symbols to represent operations that the user can do with a pointing device and a keyboard. Four for mouse clicks: click, dou-ble click, mouse down and mouse up. Four for mouse movements: mouse over, mouse out, mouse move and scroll. And four for actions on the keyboard: key click, key down and key up and typing.

The only part of the technique that needed to be extended was the symbols for user actions. This extension was done in three ways, shown on the three columns of Figure 1 and described on the following paragraph.

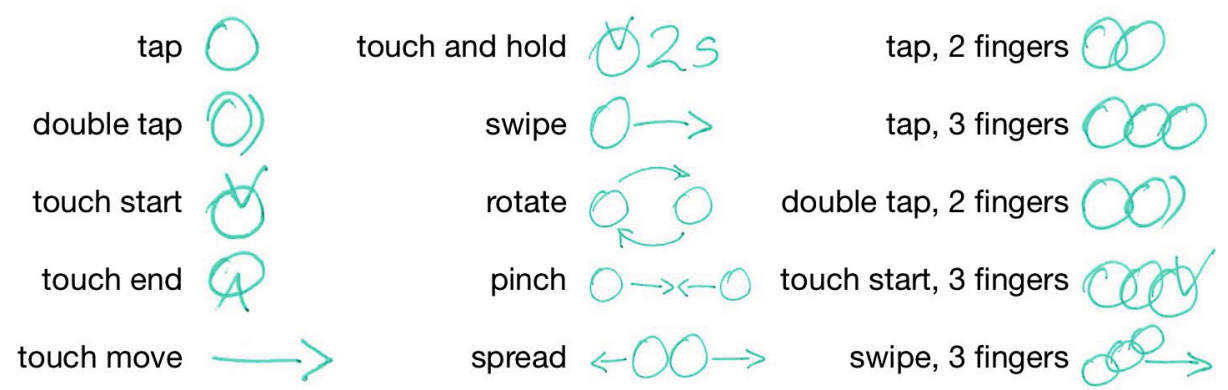

Fig. 1. Symbols for user actions on the extended version of the technique 
On the first column we have five symbols using icons that already existed on the previous version. We only changed their names to match the terminology used in multi-touch. Click became tap; double click became double tap; mouse down became touch start; mouse up became touch end; and mouse move became touch move.

On the second column we have five symbols for new actions for multi-touch inter-faces: touch and hold, swipe, rotate, pinch and spread. On the touch and hold symbol, the " $2 \mathrm{~s}$ " to the right of the touch indicates the duration of the touch, two seconds in this case.

On the third column we have multi-touch versions of the basic symbols. We indi-cate each touch (a point of contact) with a circle, so taps with two and three touches use two and three circles respectively (first two examples). We also combine the number of circles with the other symbols; for example, a double tap with two touches combines the double tap symbol with two circles (third example). The last two exam-ples demonstrate how to represent touch start and swipe with three touches.

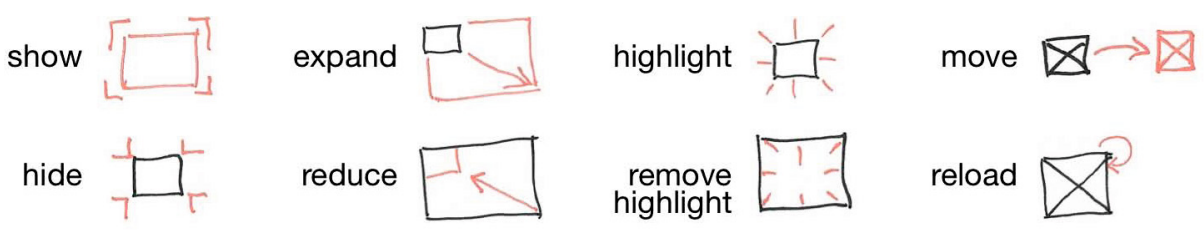

Fig. 2. Symbols for system actions, same for WIMP and multi-touch versions of the technique

Symbols for system actions didn't require any changes. Figure 2 presents the eight symbols that represent actions that the system can produce on elements presented on the screen: show, hide, expand, reduce, highlight, remove highlight, move and reload.

Three rules are the last part of our technique. 1) Draw only what changes between frames. 2) On each storyboard stay focused on just one task. 3) The goal is to express an idea, employ the technique when useful and modify it as needed.

We now provide two examples of the technique being used to describe multitouch interactions. On the two frames to the left of Figure 3 we present an example of delet-ing a message from the Mail application of an iPhone. On the first frame the user swipes over a message and the system shows a delete button. On the second frame the user taps the button and the system removes the deleted message, moving up the following messages.

On two frames to the right of Figure 3 the user selects a word on an email message, again on the Mail application of an iPhone. On the first frame the user touches and holds the touch for two seconds and the system presents a contextual menu. On the second frame the user taps the "select" option and the system highlights the selected word. 

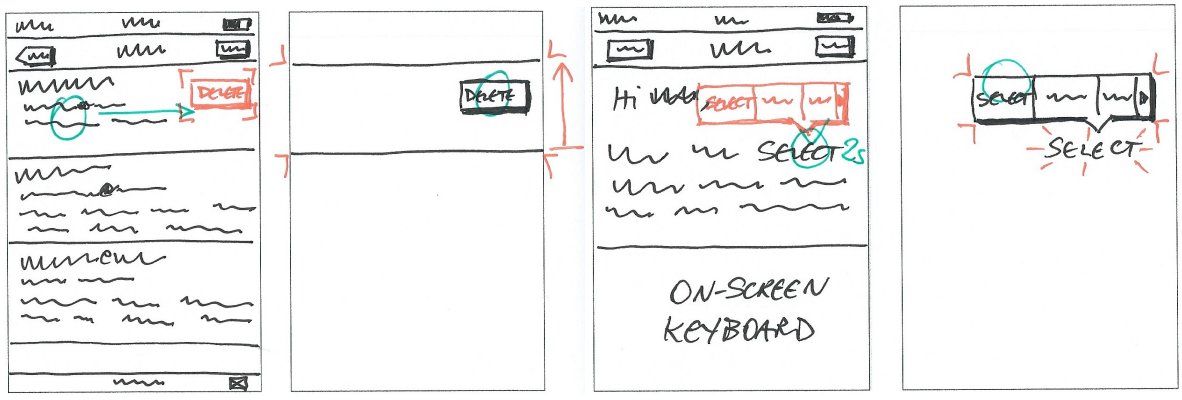

Fig. 3. Two frames on the left: representation of swipe gesture (first frame) followed by tap (second frame). Two frames on the right: representation of touch and hold (first frame) and tap actions (second frame).

\section{Conclusions}

From these examples we can see that the technique could be extended to encompass multi-touch interfaces. This encourages us to extend it further to other kinds of systems, such as gestural interfaces [9], interactive objects and embedded systems.

A preliminary feedback from professionals with background in designing touchscreen applications was very positive, and our next step is to validate it.

\section{References}

1. Agarawala, A., Balakrishnan, R.: Keepin' it real: pushing the desktop metaphor with physics, piles and the pen. In: Proceedings of the SIGCHI Conference on Human Factors in Computing Systems, pp. 1283-1292. ACM, New York (2006)

2. Apple Inc.: Cocoa Event Handling Guide. Apple Inc., Cupertino (2013)

3. Apple Inc.: Event Handling Guide for iOS. Apple Inc., Cupertino (2013)

4. Barros, G., Carneiro, G.: A technique to improve sketches of rich interactions. In: Proceedings of the 12th Brazilian Symposium on Human Factors in Computing Systems, IHC. Brazilian Computer Society, Manaus (In press) (forth-coming)

5. Buxton, B.: Sketching User Experiences: Getting the Design Right and the Right Design. Morgan Kaufmann, San Francisco (2007)

6. Cook, D.J., Bailey, B.P.: Designers' use of paper and the implications for informal tools. In: Proceedings of the 17th Australia conference on Computer-Human Interaction: Citizens Online: Considerations for Today and the Future, pp. 110. Computer-Human Interaction Special Interest Group (CHISIG) of Australia, Narrabundah (2005)

7. Johnson, G., et al.: Computational Support for Sketching in Design: A Review. Found Trends Hum-Comput Interact 2(1), 1-93 (2009)

8. Lawson, B.: How designers think: the design process demystified. Architectural Press, Oxford (2005) 
9. Saffer, D.: Designing gestural interfaces. O'Reilly, Beijing (2009)

10. Stolterman, E., et al.: Designerly Tools. Undisciplined? Design Research Society Conference 2008 (2009)

11. Verstijnen, I., et al.: Sketching and creative discovery. Des. Stud. 19(4), 519-546 (1998)

12. Wobbrock, J.O., et al.: User-defined gestures for surface computing. In: Proceedings of the SIGCHI Conference on Human Factors in Computing Systems, pp. 10831092. ACM, New York (2009) 